A puntes del CENES

ISSN 0120-3053

Volumen 30 - №. 51

Primer Semestre 2011

Págs. 45 - 59

\title{
La educación y su efecto en la formación de capital humano y en el desarrollo económico de los países
}

\section{Education and its effect on human capital formation, growth and countries' economic development}

Andrea Briceño M osquera*

Fecha de recepción: 30 de septiembre de 2010

Fecha de aprobación: 12 de noviembre de 2010

Economista y Magíster en Gobierno y Políticas Públicas, Universidad Externado de Colombia en convenio con Columbia University, Estados Unidos. Directora de Investigaciones, Facultad de Economía, Universidad Católica de Colombia. Docente Universidad Empresarial de la Cámara de Comercio de Bogotá D.C y Universidad Central.

Correo electrónico: abriceno@ucatolica.edu.co 


\section{Resumen}

El efecto dela educación en la formación de capital humano y en el desarrollo económico de los países, ha sido analizado en diversos estudios teóricos y empíricos. Los enfoques clásico de Smith y neoclásico de Solow, la teoría de capital humano planteada por Danison, Schult y Becker, y el enfoque de desarrollo y libertad de Sen entreotros, han analizado dicho efecto, evidenciando que la educación es uno de los factores determinantes de una mayor productividad, la cual se ve reflejada en la generación de nuevas tecnologías, procesos y productos, pero quizá su más significativo impacto se nota en los beneficios tanto privados como sociales que comprende, en términos de generación deingresos, inser ción en el mercado laboral, reducción de la pobreza, apropiación de los bienes culturales, y en general en el desarrollo decapacidades individuales y sociales. La formación de capital humano cobra mayor fuerza en un mundo globalizado caracterizado por constantes transformaciones económicas, tecnológicas, sociales y culturales, entre otras.

Palabras clave: educación, capital humano, desarrollo económico.

Clasificación JEL: I 25, I31, 047

\section{Abstract}

The effect of education on human capital formation and economic development of the countries, has been analyzed through theoretical and empirical studies. Classical and neoclassical theories of authors such as Smith and Solow respectively, the human capital theory by Danison, Schultz and Becker, and the theory of Sen's development among others, had analyzed the effect of education on growth and economic and social development of the countries, showing that education is one of the determinants in increased productivity, which is projected in the generation of new technologies, processes and products, but probabily, it's most significant effect are the private and social benefits, which includes, in terms of income generation, labor market integration, poverty reduction, ownership of cultural property and generally in the individual and social development. Human capital formation takes greater force in a globalized world characterized by constant economic, financial, technological, social and cultural changes, among others.

Key words: education, human capital, economic development.

JEL Classification: I 25, 131, 047 


\section{Platón}

\section{Introducción}

L a educación es un eje fundamental del desarrollo económico de una sociedad. Sobre ella recae en gran medida la posibilidad de que los países logren incrementar sus índices de innovación, productividad y crecimiento económico, permitiéndoles disminuir los niveles de pobreza einequidad. La educación, además de generar beneficios individuales, también genera externalidades sociales en la medida que permite una mayor cohesión social y beneficios intergeneracionales. Coadyuva en la creación de ingresos futuros, dado que las personas adquieren conocimientos y desarroIlan habilidades que les permiten incrementar sus capacidades productivas y de cohesión social, y de esta forma alcanzar un mayor bienestar. V ale señalar que la relación entre educación y desarrollo económico y social de los países no es automática, ni causal, puesto que una mayor y mejor educación, por sí sola, no garantiza el desarrollo económico, si no se propician algunas políticas que permitan dicho efecto.

La educación, entendida como un proceso gradual de adquisición de conocimientos y habilidades, permite potencializar las capacidades del individuo y transformarlo en un agente productivo, en la medida en que crea valor agregado y mejora su entorno. Desde esta perspectiva, "la educación se percibe como una fuerza necesaria para la adaptación a la economía globalizada, para restablecer la cohesión e integración social, alcanzar metas democráticas de largo plazo y aumentar las posibilidades de la equidad y reducción de la pobreza para el 
desarrollo del individuo, en la familia y la sociedad" 1 . A sí, la educación es el principal elemento de formación de capital humano, en la medida que determina la productividad y el bienestar de los individuos en una sociedad.

Es pertinente enfatizar que los niveles de educación (primaria, secundaria y superior o terciaria) afectan de manera diversa a las economías dependiendo de su grado de desarrollo. En este sentido, algunos estudios han concluido que los niveles de educación primaria y secundaria tienen un efecto significativo en el crecimiento de países en vía de desarrollo, y la educación superior en las economías desarrolladas². Se destaca el trabajo de U seche ${ }^{3}$ en Colombia, quien concluyó que el diferencial del $40 \%$ en el crecimiento económico entre los países del Este A siático y América L atina, a partir de la década del ochenta, es explicado por un incremento en la inversión en educación terciaria 0 superior.

El presente artículo tiene como propósito fundamental analizar el efecto de la educación en el desarrollo económico y social de los países, a partir de elementos comunes de los planteamientos clásico y neoclásico, de la teoría de capital humano, y del señalamiento de algunos resultados de estudios empíricos realizados en diferentes países.

\section{Teorías clásica y neoclásica}

A finales del siglo XVIII, A dam Smith señaló que la mayor división social del trabajo, producto de la especialización y la capacitación de la fuerza laboral, era la principal determinante de una mayor productividad, acumulación de capital y crecimiento económico, dado que la especialización en una labor u oficio, genera mayores conocimientos y cualificaciones, lo que hace más eficiente el proceso productivo. A demás planteó cómo la educación en el corto plazo podría considerarse un gasto, pero en el mediano y en el largo plazos se vería reflejaba en la productividad del trabajo y se convertiría, entonces, en una inversión. En su obra, "Naturaleza y Causa de la Riqueza de las Naciones", Smith defendió su planteamiento comparando a un hombre educado con una costosa máquina. Al respecto Ramírez, refiriéndose a Smith señaló: "Un hombre educado a expensas de mucho trabajo y tiempo, en cualquiera de aquellos oficios que requieren una

\footnotetext{
FERNANDEZ, Agustín. Análisis crítico de la relación entre educación y crecimiento económico: la Teoría del Capital Humano, (En línea). <http://www.docstoc.com/.../desafíos-de-la-educación-en-el-salvador>. [Consultado en febrero 18 de 2010].

2 PSACHAROPOULOS, George y WOODDHALL, Mauren. Educación para el desarrollo: un análisis de opciones de inversión. Publicación del Banco Mundial. Madrid (España): Tecnos, 1987. p. 42.

3 USECHE, Alejandro. Valoración del Impacto de la Metodología de Formación Teórico-Práctica: El caso de la Fundación Universitaria Empresarial de la Cámara de Comercio de Bogotá: 2009, p. 26.
} 
destreza y pericia extraordinaria, debe compararse a una costosa máquina" ${ }^{4}$. Desde esta perspectiva, la educación y la formación para el trabajo, se traducen en "capital", que debe recibir una remuneración al igual que la inversión en capital físico.

Se ha planteado que el crecimiento económico es el resultado de la acumulación del capital físico y humano, que junto con el progreso tecnológico, incrementa la productividad de dichos factores. Marshall en 1890 consideró que la educación, así como el aprendizaje en el puesto de trabajo, permitía aumentar la eficiencia industrial y formar capital. Fisher en 1920 consideró que la educación, y especialmente el fruto de ella, constituía un stock de recursos que permitía originar futuros flujos de ingresos, apreciación que permitió considerar una vez más, la formación y acumulación de conocimientos, como capital.

Es así como el capital físico y el capital humano -entendido éste último, como un stock de conocimientos, capacidades y habilidades que potencializan la productividad del ser humano-, pueden ser considerados, factores de producción, en la medida en que ambos pueden ser acumulados. Sin embargo, los capitales físico y humano tienen componentes diferenciadores, en especial la función de producción de capital físico es diferente a la de capital humano, debido a que el segundo requiere relativamente más educación y formación, entre otros, para su reproducción. En otras palabras, el capital humano es más intensivo en educación. Otra distinción importante entre capital físico y humano es que para acumular el segundo, las personas deben emplear su propio tiempo, mientras que el primero se puede comprar, heredar 0 regalar, sin necesidad de un esfuerzo propio 5 .

El crecimiento y desarrollo económico no son producto de la acumulación, por sí sola, de factores de producción como lo plantearon los clásicos, sino de la combinación eficiente de estos factores: de esta forma se genera progreso técnico. En este sentido, las tasas de crecimiento económico obedecen mucho más al ritmo al que crece la productividad de los factores productivos, que a la velocidad con la cual se acumulan. Al respecto, Schumpeter en 1912, diferenció las nociones de crecimiento y desarrollo económico. En su obra Teoría del desarrollo económico, no consideró que la acumulación de capital fuera el motor de desarrollo, así se tratara de "capital humano", sino la innovación, el

\footnotetext{
4 RAMíREZ, Duvan Emilio. Capital Humano como factor de crecimiento económico: caso departamento de Caldas (Colombia), 1983 - 2003. Caldas. Edición electrónica gratuita. Texto completo en www.eumed.net/libros/2007b/271/ 2004, p. 21-49.

5 ibid., p. 30
} 
emprendimiento empresarial y la combinación de recursos existentes, produciendo tecnología y nuevas formas de producción. A demás planteó que el crecimiento económico se evidencia en un aumento de la riqueza, mientras que el desarrollo implica diversos aspectos sociales, políticos, culturales y psicológicos. El autor destaca que en su modelo endógeno de crecimiento, la innovación tecnológica es ese elemento que los clásicos no descifraron y que constituye la solución ante el pesimista estado estacionario al que habían Ilegado.

Posterior a la Segunda Guerra M undial, el premio Nobel de Economía, Solow, en 1956, sentó las bases para explicar el modelo de crecimiento económico exógeno. Este se fundamentó en una función de producción con dos factores, capital y trabajo, y rendimientos constantes a escala. Según el modelo, el crecimiento llega a un estado estacionario en el largo plazo, que puede presentar rendimientos decrecientes a escala, porque la productividad del capital se hace menor, y sólo se logrará un crecimiento en virtud de factores exógenos. En su análisis tomó como variables explicativas del crecimiento, la inversión en capital físico y el aumento del flujo del trabajo, medidas en horas hombre, encontrando que tales factores explicaban solamente un $50 \%$ del crecimiento observado en la producción de los Estados Unidos ${ }^{6}$.

D e esta forma surgió el interrogante sobre cuáles eran las variables que explicaban el otro $50 \%$ del crecimiento. Éstas fueron consideradas en años posteriores como variables de crecimiento endógeno y estaban relacionadas con el conocimiento, la información, la capacidad empresarial y la productividad?

\section{Teoría del capital humano y su efecto económico}

En la década del 60, Danison, Schultz y Becker, fundamentados en algunos postulados de Smith y en el M odelo de Crecimiento Exógeno de Solow, formularon la Teoría del Capital Humano, planteando que la educación debe ser considerada como una inversión que realizan los individuos y que les permite aumentar su dotación de capital humano, que tanto o más que la compra de bienes de capital, aporta de manera determinante al crecimiento económico de los países, en virtud de su nexo directo con la productividad8. En sus estudios empíricos, estos autores mostraron cómo

6 ÁVILA, Óscar Iván. Desigualdad, educación y crecimiento económico. Bogotá D.C., 2009, p. 15. Tesis de Maestría Universidad del Rosario (Facultad de Economía) p. 15.

7 Los estudios pioneros de Solow en este campo son: A Contribution to the Theory of Economic Growth, En: Quarterly Journal of Economics, No. 70, 1956, p. 65-94, y Technical Change and the Aggregate Production Function. En: Review of Economics and Statistics. No. 39, 1957, p. 312-320.

8 Los fundamentos de esta teoría fueron expuestos en: BECKER, G. Investment in Human Capital:A Theoretical Analysis. En:The Journal of Political Economy, No. 10, 1962, p. 9-49, y Shultz, T. Investment in Human Capital. En: The American Economic Review, No. 51, 1961, p. 1-17. 
los conocimientos y las habilidades adquiridas con el estudio y la experiencia, son factores esenciales del crecimiento y explican en gran parte las diferencias en el ingreso per cápita entre países.

Danison anal izó cómo, entre 1910-1960, el crecimiento económico de Estados Unidos, A rgentina, M éxico y Brasil, se debió al incremento de la educación de la fuerza de trabajo en un $23 \%, 16 \%$, $1 \%$ y $3 \%$, respectivamente 9 . Schultz también realizó un análisis similar, pero calculando el stock de educación de la fuerza de trabajo y su rendimiento para conocer cómo contribuía al aumento del ingreso nacional. En su estudio para el periodo 1929-1957, el incremento de la educación explicó entre el $16,5 \%$ y el $20 \%$ del crecimiento del ingreso nacional en Estados U nidos. A sí mismo, a partir de la reflexión sobre la necesidad de destinar recursos a la inversión en educación y como un aporte de la teoría del crecimiento, planteó:

Los factores de producción decisivos para el mejoramiento del bienestar de los pobres no son el espacio, la energía y la disponibilidad de tierra cultivable; los factores decisivos son el mejoramiento de la calidad de la población y en este sentido, la formación educativa y los adelantos en el conocimiento son determinantes ${ }^{10}$.

Desde el argumento de destinar recursos al gasto en educación, vale la pena mencionar que, Barro, profesor de la Universidad de Harvard, quien realizó un estudio sobre el efecto dela educación en las tasas de crecimiento del producto per cápita en 100 países, en el periodo 1965-1995, estimó que un año adicional de educación superior eleva en casi 0.5 puntos porcentuales la tasa de crecimiento de las economías en países en vía de desarrollo ${ }^{11}$.

Becker, premio Nobel de Economía y, como se mencionó, uno de los autores de la Teoría del Capital Humano, afirmó:

El continuo crecimiento en los ingresos per cápita de muchos países durante los siglos XIX y XX es en parte debido a la expansión del conocimiento científico y técnico que incrementa la productividad del trabajo y de otros factores de la producción... Y la creciente dependencia de la industria en el conocimiento sofisticado realza de gran manera el valor de la educación, la educación técnica y el entrenamiento en el sitio de trabajo ${ }^{12}$.

\footnotetext{
9 PSACHAROPOULOS, George y WOODDHALL, Maureen. Op. cit., p. 27.

10 RAMÍREZ, Op.cit., p. 6.

11 BARRO, John. Human Capital and Growth. En: American Economic Review. Papers and Proceedings, Vol. 91, No. 2, 2001, p. 52.

12 BECKER, Gary S. Human Capital: A Theoretical and Empirical Analysis, with Special Reference to Education. New York: Columbia University Press for NBER. $2^{\text {nd }}$ Ed., 1964, p.36.
} 
Pocos países o quizá ninguno han alcanzado un período de crecimiento económico sostenido sin inversiones importantes en su fuerza de trabajo.

Otro estudio destacado sobre la relación entre educación y crecimiento económico fue el que realizó M ushkin en Estados Unidos, quien consideró que para entender la importancia de la educación como un factor en la formación de capital, es útil pensar en la analogía de cómo los recursos naturales de una nación, no pueden por sí mismos y sin mejoras conducir a productos finales con un alto valor agregado. Sin embargo, luego de hacer parte de una función de producción con capital y trabajo, producen cosechas, minerales, capital y energía. En palabras de Mushkin: "No hay un término genérico para los esfuerzos de desarrollo que deben ser aplicados a los recursos naturales con el fin de convertirlos en capital productivo, pero sí hay un término para describir los esfuerzos para desarrollar los recursos humanos: es educación"13. I gualmente hace un análisis sobre los requerimientos de educación para lograr el crecimiento económico, poniendo en evidencia el hecho de que no solamente es importante aumentar la inversión en educación, y con esta la cobertura, sino que ella debe ser orientada hacia aquellos trabajos que generen mayor productividad y bienestar social.

Delfino señaló que Hicks en la década del 80, estudió la relación entre analfabetismo y crecimiento económico, como una medida de desarrollo educativo en el periodo 1960 - 1977, en 83 países en desarrollo y encontró que en 12 países las más altas tasas de crecimiento económico tenían un promedio menor de analfabetismo y mayores expectativas de vida14.

De acuerdo con el estudio de Delfino, el autor señala que con el mismo propósito de demostrar el efecto de la educación en el crecimiento económico, Wheeler en 1980 diseñó un model o que evidenció cómo la educación contribuyó al crecimiento del Producto Interno Bruto en Estados U nidos, no sólo directa, sino indirectamente y aumentó la tasa de inversión. En este sentido, el estudio arrojó que un incremento de la inversión en capital humano del $20 \%$ al $30 \%$, causó un aumento de la inversión en capital físico entre el $8 \%$ y $16 \%$. A sí mismo, resalta que Easterlin concluyó para 25 países, que el despliegue tecnológico y el crecimiento económico moderno dependía del mayor potencial de aprendizaje y de la motivación que provenía del desarrollo de la educación formal ${ }^{15}$.

\footnotetext{
13 MUSKIN, Selma J. Economics of Higher Education. Washington: US. Govt.Print Off, 1962, p. 85-92.

14 DELFINO, José A. Educación y progreso económico y social. En: Revista Actualidad Económica, Año XII, № 53, agosto-diciembre de 2002, p. 30.

15 Ibid., p. 45.
} 
Los economistas Paul Romer y Robert L ucas demostraron por medio de análisis empíricos, cómo el capital humano era una variable endógena y no exógena, como lo planteó el modelo neoclásico de Solow ${ }^{16}$. Desde el enfoque de estos autores, el capital humano determina la creación de nuevos procesos, tecnologías y productos, y por ende se constituye en el impulsor del desarrollo económico en los países.

Desde este punto de vista el capital humano utiliza el capital físico y la tecnología de manera más eficiente, explicando un modelo de crecimiento endógeno, que genera nuevas y mejores formas de producción. M ás aun, la mano de obra capacitada aumenta la eficiencia global del trabajo.

Romer, en 1990, comparte en sus planteamientos los postulados de Smith, a propósito de la división del trabajo y de su especialización, y considera que el crecimiento es el resultado de la acumulación de conocimientos específicos y resalta que, aunque los diferentes conocimientos comparten la propiedad de no ser excluyentes, son heterogéneos, por lo cual la exclusividad en su uso depende, tanto de la propia formación impartida, como de las instituciones que regulan los derechos de propiedad.
Lucas, Premio Nobel de Economía en 1995, comprobó la existencia de rendimientos crecientes para el capital humano. En este sentido, indicó que las diferencias entre países tienden a acentuarse debido a que con rendimientos crecientes, el capital humano estará más remunerado en los países con una mayor dotación inicial, razón por la cual trabajadores cualificados tenderán a desplazarse hacia los países con mayores salarios. En 2001 Echart y Sumel demostraron que la existencia de rendimientos crecientes asociados a la especialización y a la división del trabajo, en el sector industrial, generaban crecimientos económicos desiguales entre países, donde se da lo que se denomina un «círculo vicioso» de crecimiento ${ }^{17}$.

De acuerdo con Villa, en la década del noventa $M$ ankiw y Weil diseñaron un modelo que consideró la inversión en educación de 98 países para el periodo 1960-1985 y halló que las diferencias en las tasas de ahorro, educación y crecimiento poblacional, explican las discrepancias entre los PIB per cápita de los países. Es de destacar que en 1957, Corea del Sur contaba con un ingreso por habitante equivalente al de G hana, para que en un plazo de 30 años, contara con un ingreso seis veces mayor; tal nivel impresionante de

\footnotetext{
16 Recuérdese, como se señaló anteriormente que la teoría neoclásica sostenía que el crecimiento económico era el resultado de la acumulación de capital físico y de la ampliación de la fuerza de trabajo, combinados con un factor exógeno como era el progreso tecnológico, que incrementaba la productividad del capital y de la fuerza del trabajo.

17 ECHART, María, SUMEL, Nuria y PANTANO, Juan. Rentabilidad de la educación, Buenos Aires: Fiel, 2001, p. 25-55.
} 
crecimiento se debió, al menos en un $50 \%$, al éxito logrado en la formación educativa y en la aplicación de conocimientos específicos ${ }^{18}$.

A I analizar el diferencial del $40 \%$ en el crecimiento económico, entre los países del Este Asiático y A mérica Latina, a partir de la década del 80, U seche explicó el fenómeno en razón a un incremento en la inversión en educación terciaria o superior. Corea del Sur y Singapur lograron en poco tiempo un crecimiento económico sostenible y aumentaron su producto interno bruto (PIB) per cápita en nueve y siete veces, respectivamente, con altos niveles de competitividad, entre 1965 y $1996^{19}$.

A I analizar los argumentos a propósito de los elementos que influyen en los individuos para invertir en educación, Corazzini se concentró en el estudio de los factores que determinan la decisión de los agentes para continuar su educación formal en el nivel superior, mediante la estimación implícita del valor presente de los beneficios de asistir a ella y su comparación con el valor presente tanto de los costos directos como del costo de oportunidad que implica la decisión ${ }^{20}$.
A I respecto, la rentabilidad de la educación puede ser cal culada como la tasa interna de retorno resultante de la relación entre los costos directos e indirectos de invertir en educación y las trayectorias de ingresos laborales que los individuos obtienen a lo largo de su vida activa, para cada nivel educativo dado. Autores como Psacharopoulos sostienen que la tasa social de retorno incluye los costos y los beneficios que la sociedad y el Estado, producen y obtienen en el proceso educativo ${ }^{21}$.

En Colombia, Sánchez ${ }^{22}$ analizó las tasas de retorno de la educación como un determinante de las decisiones de los individuos al formarse y del Estado al formular la política pública en educación. Estimó que por cada año de estudio de un individuo, se espera que su ingreso mejore en aproximadamente el $12.85 \%$. De igual forma, se espera que por cada año de experiencia se obtenga un $4.87 \%$ de crecimiento en el salario. Es de destacar que, según este estudio, la rentabilidad de la educación superior para Colombia es del $17 \%$. Es notorio que la educación tiene una mayor influencia en el crecimiento del salario

$\overline{18}$ VILLA, Leonardo. Economía de la educación. Bogotá: Universidad de los Andes, 2001, p.16.

19 USECHE, Alejandro. Valoración del impacto de la metodología de formación teórico-práctica: el caso de la Fundación Universitaria Empresarial de la Cámara de Comercio de Bogotá: ccb, 2009, p. 26.

20 CORAZZINI, A, DUGAN, D y GRABOWSKI, H. Determinants and Distributional Aspects of Enrollment in U.S. Higher Education. New York. Publicado por The Journal of Human Resources, 1972, p. 39-59.

21 PSACHAROPOULOS, George. The Profitability of Investment in Education: Concepts and Methods (en línea). <http:/ /www.worldbank.org/html/extdr/hnp/hddflash/workp/wp_00063.html>. Washington US. Editorial Banco Mundial 1995. p. 2 -15. [Consultado el 25 de mayo de 2010].

22 SÁNCHEZ Rengifo, Hernán. Rentabilidad de la inversión en educación en la ciudad de Cali (la función minceriana y el método elaborado). En: Revista Asuntos Económicos, Calí Centro de Estudios Económicos y Administrativos, 2004, p. 35. 
y en el posicionamiento y la movilidad social.

A dicionalmente, los individuos con un determinado nivel de capital humano son más productivos si se rodean de otros con uno mayor. Con esto se generan externalidades positivas del conocimiento. Este planteamiento está fundamentado en un estudio sobre el crecimiento de los países industrializados del sudeste asiático, en el cual se resal ta que la principal fuente del crecimiento es la acumulación de capital humano y el aprendizaje en el puesto de trabajo, por encima de la inversión en capital físico ${ }^{23}$.

Es de destacar que los capitales físico y humano no son suficientes para lograr el crecimiento económico, porque debe existir, paralelamente, una estructura institucional sólida para explotarlo. A sí la educación es considerada como una inversión por quienes se esfuerzan en obtenerla; es un medio para lograr mayores ingresos y prestigio social. Sin embargo, países con una amplia oferta en educación, que no generen las condiciones funcionales y laborales para los individuos formados, se verán enfrentados, por una parte, a grupos frustrados que pueden generar agitaciones políticas y sociales, y por otra parte, a fuga de "cerebros", debido al desacuerdo de ingresos y posición social que creen merecer en atención a sus estudios.

\section{C apital humano y desarrollo social}

A finales del siglo pasado, en 1998, Sen Premio Nobel de Economía, reafirmó la importancia de la educación como uno de los principales determinantes del crecimiento y desarrollo económico de los países, pero centró su análisis en la explicación de cómo la educación constituye un medio para ampliar las capacidades y posibilidades de los individuos, y de esta forma mejorar la cal idad de vida. D esde esta perspectiva, el capital humano, y mejor aún, la capacidad humana, se pueden considerar como expresiones de libertad para tomar decisiones económicas, sociales, políticas y culturales, entre otras.

Si la educación aumenta la productividad de las personas en la producción de bienes y servicios, y en la generación de valor agregado a los mismos, se trata de una mejora en el capital humano, que puede aumentar el nivel de ingresos de la persona educada; pero al mismo tiempo, le proporciona otros beneficios, como el poder comunicarse y desarrollarse integralmente en otras actividades que contribuyen a su bienestar. En efecto, los beneficios de la educación van mucho más allá de la producción de bienes. Se comparte el planteamiento de Sen al señalar que: " $\mathrm{Si}$ una persona, mejorando su educación, su salud y otros aspectos, puede ser más productiva en la fabricación de bienes,

\footnotetext{
$\overline{23}$ USECHE, Op.cit., p. 26.
} 
es lógico esperar que gracias a estos medios también tenga más posibilidades y libertad para desarrollar una mejor calidad de vida" ${ }^{24}$.

No debe obviarse que la educación, además de generar beneficios económicos privados, -como se ha intentado demostrar a lo largo de este capítulo, con base en la revisión teórica y el análisis de resultados de modelos y análisis empíricos-, también genera beneficios sociales. Según Villa ${ }^{25}$, algunos de los beneficios sociales de la educación se reflejan en la reducción de la criminalidad, la mayor cohesión social, las innovaciones tecnológicas y los beneficios intergeneracionales, entendidos como las externalidades positivas que generan sobre sus hijos los padres con un mayor nivel de formación educativa. Un individuo con mayores niveles de educación reduce la probabilidad de estar desempleado e incrementa su nivel de ingresos, pues es un trabajador más productivo, y consecuentemente, un menor desempleo disminuye los efectos negativos de los crímenes y la violencia.

Según Á vila ${ }^{26}$, los retornos privados de la educación, y en general supuso que los retornos social es de la educación, son superiores. El argumento detrás de esta conclusión es que la educación permite armonizar las relaciones sociales, mejorar la calidad de vida y aumentar la participación ciudadana, lo cual tiene un efecto positivo sobre el crecimiento y el desarrollo económico y social de los países.

D ebe rechazarse la concepción de limitar la educación a un instrumento de preparación para un orden laboral existente, dando mayor importancia a un proceso formativo de valores. En este sentido, el sistema educativo no puede limitarse al servicio del sector productivo, sino que además tiene la función de incrementar la eficiencia social. Para defender este argumento es pertinente señalar la hipótesis del filtro planteada por el Banco M undial en 2006, según la cual la educación no sólo desarrolla habilidades y conocimientos vocacionales, sino que afecta igual mente las actitudes, motivaciones y valores que, sumados, determinan la productividad de los individuos y sus posibilidades de ser empleados. De acuerdo con esta hipótesis: "La educación superior provee conocimientos específicos y desarrolla habilidades de razonamiento, también induce cambios en las creencias, los valores y las actitudes hacia el trabajo y la sociedad" 27.

\footnotetext{
${ }_{24}$ SEN, Amartya. Desarrollo y Libertad. Bogotá: Planeta, 2001, p. 125.

25 VILLA, Leonardo. Op. cit., p.16.

26 ÁVILA, Óscar Iván. Desigualdad, educación y crecimiento económico. Bogotá, 2009, p. 32. Tesis de Maestría Universidad del Rosario (Facultad de Economía).

27 BANCO MUNDIAL. La Educación Terciaria en Colombia, Washington US: OECD 2008, p. 53.
} 
Finalmente es pertinente señal ar algunas posturas críticas de diversos autores, a propósito del efecto de la educación en la formación de capital humano. En efecto, D oeringer en 1971, con su teoría de los mercados internos y el dualismo, y Piore en 1975 , con su teoría de los mercados segmentados, consideran que es únicamente la experiencia laboral la que tiene un efecto en la formación de capital humano, y no la educación recibida. A demás, cuestionan la supuesta relación entre educación e ingresos, en virtud de que estos últimos no sólo dependen de la cualificación de los individuos, sino de una serie de instituciones, normativas y relaciones de negociación, que los determinan.

\section{Conclusiones}

El efecto de la educación en el desarrollo económico y social de los países, ha sido estudiado por innumerables autores, quienes han demostrado por medio del análisis teórico y comprobación empírica, dicho efecto. L os enfoques clásico (Smith - 1776) y neoclásico (Solow - 1956) plantearon cómo la educación tiene un efecto en la acumulación del capital físico y humano, que junto con el progreso tecnológico, incrementa la productividad de dichos factores. A principios de la década del 60, Danison, Schultz y Becker, formularon la Teoría del Capital Humano, planteando que la educación debe ser considerada como una inversión que realizan los individuos y que les permite aumentar su dotación de capital, que tanto o más que la compra de bienes, aporta de manera determinante al crecimiento económico de los países, en virtud de su nexo directo con la productividad. En este contexto, diversos estudios referenciados en este artículo, han demostrado cómo el crecimiento económico de muchos países durante el siglo $X X$ fue en parte debido a una mayor y mejor educación, y con esta, la expansión del conocimiento científico y técnico que incrementa la productividad del trabajo y de otros factores de producción.

A finales del siglo pasado, el Premio Nobel de Economía, A martya Sen, reafirmó la importancia de la educación como uno de los principales determinantes del desarrollo económico de los países, pero centró su análisis en la explicación de cómo la educación constituye un medio para ampliar las capacidades y posibilidades de los individuos, y de esta forma mejorar la calidad de vida de la sociedad.

Es claro que la educación coadyuva en la creación de ingresos futuros, en la medida en que las personas desarrollan habilidades y conocimientos que les permiten incrementar sus capacidades productivas y sociales, y de esta forma generar mayores ingresos y riqueza, alcanzando un mayor bienestar y cohesión social, además de reducir la criminalidad. 


\section{R eferencias Bibliográficas}

1. ALTBACH, PhilipG., REISBERG, Liz y RUMBLEY, Laura E. Trends in Global Higher Education: Tracking an A cademic Revolution. A Report Prepared for the UNESCO 2009 W orld Conference on Higher Education. New Y ork, 2009, p. 122.

2. Á VILA, Óscar Iván. Desigualdad, educación y crecimiento económico. Bogotá, 2009, p. 32. Tesis de M aestría Universidad del Rosario (Facultad de Economía).

3. BANCO MUNDIAL. La Educación Terciaria en Colombia, W ashington US: OECD 2008, p. 53.

4. BARRO, John. Human Capital and Growth. En: A merican Economic Review. Papers and Proceedings, $\mathrm{V}$ ol. 91, No. 2, 2001, p. 52.

5. BECKER, Gary S. Human Capital: A Theoretical and Empirical A nalysis, with Special Reference to Education. New York: Columbia University Press for NBER. $2^{\text {nd }} E d ., 1964$, p. 55.

6. CORAZZINI, A, DUGAN, D y GRABOWSKI, H. Determinants and Distributional A spects of Enrollment in U.S. Higher Education. New Y ork. Publicado por The Journal of Human Resources, 1972, p. 39-59.

7. DELFINO, José A. Educación y progreso económico y social. En: Revista A ctualidad Económica, A ño XII, Nㅇ 53, agosto-diciembre de 2002, p. 30.
8. DOERINGER, P. y PIORE, M. 1971, Internal $L$ abor $M$ arkets and $M$ anpower A nalysis. (En línea). http://ddd. uab. cat/ pub/papers/02102862n55p45.pdf. [Consultado el 25 de mayo de 2010].

9. ECHART, María, SUMEL, Nuria y PANTANO, Juan. Rentabilidad de la educación, Buenos Aires: Fiel, 2001, p. 25-55.

10. FERNÁN DEZ, A gustin. A nálisis crítico de la relación entre educación y crecimiento económico: la Teoría del Capital Humano, (En línea). < http:// www. docstoc. com/.../desafios-de-laeducación-en-el-salvador> . [C onsultado en febrero 18 de 2009].

11. LUCAS, R. E., Jr. On the mechanics of economic development. Journal of M onetary Economics 22, 3-42, 1988.

12. MARSHALL A. Economics, in Relation to the $M$ an and to His Times. In The American Economic Review Vol. 31, No. 2 (Jun., 1941), p. 223-235.

13. MUSKIN, Selma J. Economics of Higher Education, Washington US. Govt.Print Off, 1962, p. 85-92

14.PSACHAROPOULOS, George y WOODDHALL, Mauren. Educación para el desarrollo: un análisis de opciones de inversión. Publicación del Banco M undial. Madrid (E spaña): Tecnos S.A., 1987. p. 42.

15. RAM ÍREZ, Duvan Emilio. Capital 
Humano como factor de crecimiento económico: caso departamento de Caldas (Colombia), 1983 - 2003. Caldas. Edición electrónica gratuita. Texto completo en www.eumed. net/libros/ 2007b/271/ 2004, p. 21-49.

16. ROM ER, P. Capital A ccumulation in the Theory of Long-run Growth. In R obert Barro (ed.), M odern Business Cycle Theory. 1986 Cambridge : Harvard University Press, 51-127.

17 SÁNCHEZ, Rengifo, Hernán. Rentabilidad de la inversión en educación en la ciudad de Cali (la función minceriana y el método elaborado). En: Revista A suntos Económicos, Cali Centro de Estudios Económicos y Administrativos, 2004, p. 35.

18. SEN, A martya. Desarrollo y Libertad. Bogotá: Planeta, 2001, p. 125.

19. SCHUM PETER, Joseph. Teoría del desenvolvimiento económico. 5a reimp. M éxico: Fondo de Cultura Económica, 1978. p. 128 - 215.
20. SM ITH, A. A I Inquiry into the $\mathrm{N}$ ature and Causes of the Wealth of $N$ ations (eds.) R. H. Campbell y A. S. Skinner, Oxford: Oxford University Press, 1976.

21. SOL OW, Robert. A Contribution to the Theory of Economic Growth. En: Quarterly J ournal of E conomics. N o. 70, (1956), p. 65-94, y Technical Change and the A ggregate Production F unction. En: Review of E conomics and Statistics, No. 39, (1957), p. 312-320.

22. SCHULTZ, T. La Inversión en Capital Humano. Educación y Sociedad, volumen 8, № 3. 1983 p. 26-85.

23. USECHE, Alejandro. Valoración del impacto de la metodología de formación teórico-práctica: el caso de la Fundación U niversitaria E mpresarial de la Cámara de Comercio de Bogotá: C cb, 2009, p. 26.

24. VILLA, Leonardo. Economía de la Educación. Bogotá: U niversidad de los Andes, 2001, p. 16. 